\title{
Über einige histologische Ergebnisse der experimentellen Krebsforschung.
}

\author{
Von \\ Prof. Dr. Hugo Apolant.
}

Mit is Textfiguren.

Die Entwicklung der experimentellen Krebstorschung daticrt von dem Augenblick, da es gelang, echte maligne T'umoren inuelhalb der Spezies von Tier auf Tier zu transplantieren und in Generationen fortzuzüchten. Sind einmal die ersten, gewöhulich schwierigsten Passagen gegiuickt. und ist der Geschwulststamm in das Stadium der zuerst von Ebrlich beobachteten und allseitig bestätigten sogenannten Virulenzsteigermug getretell. so scheint ein spontanes Erlöschen der Wuchsenergie aus inneren. den Tumorzellen inhärenten Gründen bei Ausschluss accidenteller Momente, wie Infektion, Wahl ungeeigneter Tiere etc. nicht mehr vorzukommen. So hat $\%$. B. der schnellst wachsende Tumor unserer Abteilung in $7^{1 / 2}$ Jahren die 240. Impfgeneration erreicht. olne im mindesten in seiner Proliferationskraft nachzulassen.

Die Bedeutung derartiger unbegrenzt weiter züchtbarer Geschwulststämme liegt nun aber keineswegs nur darill, dass sie das notwendige Material für experimentelle, insbesondere immunisatorische und kurative Studien abgeben. Gewiss werden diese Bestrebungen stets unsere wichtigste und vornehmste Aufgabe bleiben. Aber es darf nicht ausser Acht gelassen werden, dass sich an die Impftumoren auch eine Fülle rein morphologischer Probleme knüpfen, und dass sich die Forschung in dieser Richtung als überaus erfolgreich erwiesen hat. Wärend wir bei den menschlichen Geschwülsten auf das Studium einzelner Stadien beschränkt sind, die uns nur in besonderen Fällen, wie etwa bei der Untersuchung von Rezidiven nach Operationen und eventuell noch durch die Sektion gewonnenen Materials einen ungefahren Einblick in die Geschichte des betreffenden Tumols gestatten, können wir bei der anscheinend unbegrenzten Lebensdaner eines Geschwulststammes die auftretenden Veränderungen ganz anders verfolgen und vor allem auch ihre Beziehungen $z u$ veründerten 
Wuchsbedingungen feststellen. Es ist daher verständlich, dass die zuerst auf experimentellem Wege aufgedeckten strukturellen und biologischen I'umorvariationen auch in der menschlichen Pathologie einem gesteigerten Interesse begegnen.

Ich habe bereits im Jahre $1906^{1}$ ) auf Grund eines Materials von 276 Einzeltumoren, das sich inzwischen auf weit über 600 vermehrt hat, eine ganz auffallende strukturelle Variabilitit der spontan entstandenen Mäusegeschwülste nachweisen liönnen. Die zahlreichen von mir aufgestellten Typen gehen ohne scharfe Grenze ineinander über und werden nicht nur in verschiedenen fieschwülsten des gleichen Tieres, sondern sogar in ein und demselben 'Tumor angetroffen, der zum T'eil papillär, zum Teil alveolär' und wieder an anderen Stellen rein acinös gebaut sein kann.

Häufiger noch als innerhalb einer einzelnen Geschwulst. aber prinzipiell in gleicher Weise, treten derartige Inderungen des Baues bei der fortgesetaten Züchtung eines Stammes auf. Von Murray") werden sie als der Ausdruck cyklischer Anderungen der Zellvitalitat angesehen, die sich nach $B$ as hford auch in periodischen Schwankungen der Wuchsenergie und Impfausbente kundgeben sollen. Ich habe mich nie von der Existenz derartiger regelmissiger Perioden ïberzeugen können und bin daher geneigt, die Wucherungstorm nicht ausschliesslich auf die vitalen Verhaltnisse der 'Tumorzellen $z u$ beziehen, sondern daneben noch als wesentlichen Faktor die sogenannte Geschwulstresistenz des geimplten Tieres gelten zu lassen, die eines der wichtigsten und meist studierten Probleme der experimentellen Krebsforschung bildet. $\% u$ dieser Auffassung veranlasste mich die mehrfach gemachte Beobachtung, dass partiell immunisierte Tiere gelegentlich einen plötzlichen Rückschlag der plexiformen Karzinomstruktur in die acinöse erkennen lassen, wie er mir unter anderen Verhältnissen nie begegnet ist.

Ich beabsichtige nicht, auf diese bereits viel diskutierte Frage hier weiter einzugehen, sondern möchte mich auf die Besprechung der sehr eigenartigen Veränderungen beschränken, die ich bei unserem Karzinomstamm 11 beobachtet habe. Einen

1) Die epithelialen Geschwülste der Maus, Arbeiten aus dem Kgl. Inst. f. experin. Ther., 1906, Heft 1.

2) Die Beziehungen zwischen Geschwulstresistenz and histologischem Bau transplantierter Mäusetumoren. Berliner klin. Wochenschr., 1909, Nr. 33. Arehiv f. mikr. Anat. Bd. $7 \mathrm{~s}$. 
kurzen Bericht über dieselben gab ich bereits auf der Kieler Tagung der Deutschen Pathologisehen Gesellschaft im Jahre 1908. Es dürfte jedoch wegen der prinzipiellen Bedeutung der hier auftauchenden Fragen nicht ohne Interesse sein, die weitere Geschichte dieses Geschwulststammes kennnen zu lernen.

Zum besseren Verständnis ist ein kurzer Überblick über eine Geschwulstveränderung unabweislich, die unzweifelhaft einen der interessantesten der auf diesem Gebiet experimentell erhobenen Befunde bildet, nämlich die sekundäre Sarkomentwicklung.

Im Jahre $1905^{1}$ ) beschrieben Ehrlich und ich einen Geschwulststamm bei der Maus, der neun Generationen hindurch als reines Karzinom gewachsen war. Plötzlich traten in dem ursprünglich sehr spärlichen Stroma lebbafte Wucherungsvorgänge auf, die Bindegewebszellen nahmen vollständig den Charakter spindliger Sarkomzellen an, und der 'Tumor bot dann mehrere Generationen bindurch das Bild einer ausgesprochenen Mischgeschwulst, und zwar speziell des von v. Hansemann ausführlich beim Menschen beschriebenen Carcinoma sarcomatodes (Fig. 1). Das

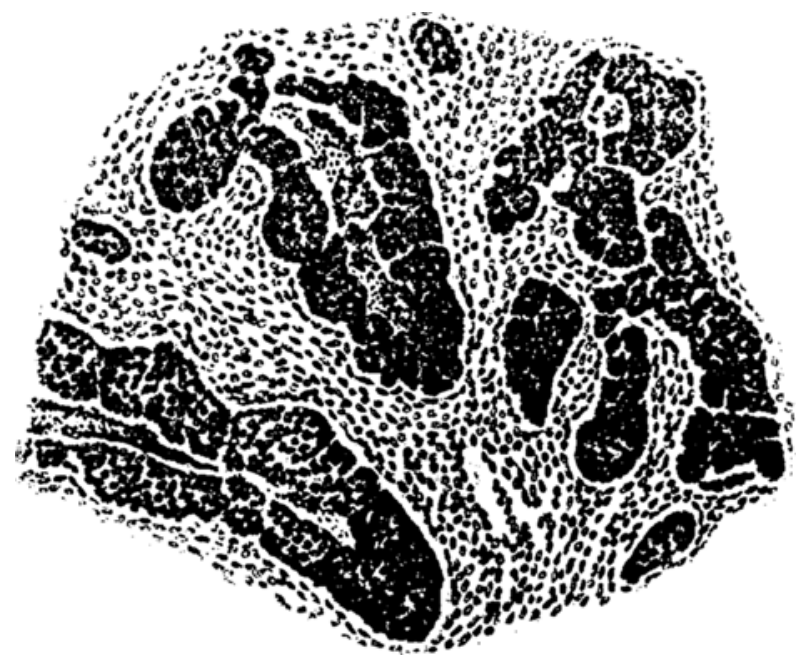

Fig. 1.

sarkomatöse Stroma nahm nun weiterhin auf Kosten der immer kleiner werdenden Karzinominseln mächtig zu, ein Prozess, der

1) Beobachtungen über maligne Mäusetumoren. Berliner Wochenschr., 1905, Nr. 28. 
schon in der 13. Generation zu einer Eliminierung der karzinomatösen Komponente führte, so dass der Tumor von nun an ein reines Spindelzellensarkom darstellte, an dem in der Folgezeit keine strukturellen Veränderungen meur auftraten.

Diese merkwürdige Beobachtung wurde anfangs sehr skeptisch aufgenommen, bis von mebreren Seiten überraschend schnell Bestatigungen eintrafen. Wir selbst beobachteten denselben Prozess, wenn auch unter Bildung eines etwas abweichend gebauten mehr polymorphzelligen Sarkoms, in zwei anderen Stämmen, während in kurzer Zeit analoge Befunde von Leo Löb, Bashford und Haaland, Liebmann, Stalır, Lewin (von letzterem bei einem Rattensarkom), ferner von ('l u n et u. a. veröffentlicht wurden. Interessanterweise mehren sich in neuerer Zeit die Publikationen über ganz gleich zu beurteilende Fălle aus der menschlichen Pathologie. Neben der mir seinerzeit zur Veröffentlichung überlassenen, viel zitierten Beobachtung $S \mathrm{chm}$ orls (anfängliches Thyreoidea-Adenom, dessen Rezidir ein Carcinoma sarcomatodes darstellte, wahlrend bei der Selition nur Sarkon gefunden wurde), nenne ich u. a. dell wahrscheinlich auch hierher gehörigen, auf dem Chirurgenkongress 1!008 mitgeteilten l'all von Senger, sowie eine Beobachtung Coenens. ${ }^{1}$ )

In der Dentung der sekundiren Sarkomentwicklung hatten Ehrlich und ich von Anfang an den Standpunkt eingenommen, dass sowobl eine metaplastische Umwandlung von Karzinom- in Sarkomzellen, als anch die Annahme einer primaren Mischgeschwulst a limine abzuweisen sind. Erfreulicherweise herrscht über letzteren Punkt völlige Einigkeit, und binsichtlich des ersteren wenigstens unter den Autoren, die über den Gegenstand eigene Erfahrungen an Tiergeschwiilsten besitzen. Dagegen habe ich speziell gelegentlich der Kieler Verhandlungen der Deutschen Pathologischen Gesellschaft, in denen diese Fragen sehr eingehend besprochen worden sind, aus privaten Bemerkungen den Eindruck gewonnen, dass von manchen Vertretern der pathologischen Anatomie die Möglichkeit einer metaplastischen Umwandlung der Karzinom- in die Sarkomzelle nicht als ganz ausgeschlossen betrachtet wird. Ohne auf die theoretischen Bedenken einer solchen Deutung hier eingehen zu wollen, möchte ich nur

1) Beiträge zur klin. Chirurg., Bd. 68, Heft 3, 1910. 
auf die gegen dieselbe sprechende histologische Tatsache hinweisen, dass nirgends auch nur die Andeutung eines direkten Überganges der einen Zellart in die andere vorhanden ist. Die Grenzen sind überall so scharf wie möglich (Fig. 1). Wie ich schon früher dargelegt habe, erinnern die Bilder vielfach ausserordentlich an die Strukturverhältnisse, die man bei der Radiumheilung der Mäusekarzinome trifft. Nur sind es in let\%terem Falle nicht spindlige Sarkomzellen, sondern die ihnen morphologisch ahnlichen vollsaftigen Fibroplasten, die in die Krebsalveolen hineinwuchern und die epithelialen Nester allmählich zum Schwund bringen.

Nach der von Ehrlich und mir gegebenen und wohl allgemein akzeptierten Erklarung beruht die sekundäre Sarkomentwicklung auf einer von den chemisch irgendwie veränderten Krebszellen ausgehenden Reizwirkung. Dass die Krebszellen ganz allgemein auf die Bildung des Stromas bestimmenden Eintluss ausüben, ist bekannt und ergibt sich allein schon daraus. dass der histologische Bau der Metastasen, für deren Eutstehung die Annahme einer Parenchymzellenverschleppung ohne Stromaelemente genügt, in allen wesentlichen Pnnkten mit dem des Primärtumors übereinzustimmen ptlegt. Ebenso aber, wie in der Metastase das Stroma nicht aus verschleppten /ellen, sondern aus den lokal vorhandenen bindegewebigen Elementen abruleiten ist, ebenso wird nach. Jensen und Bas lifol'd das Stroma der Imptkrebse vom Wirtstier geliefert. Die transplantierten Bindegewebszellen gehen vollkommen zugrunde. Hierans ergibt sich die wichtige Tatsache, dass nach unserer Theorie die Sarkomzelle nicht von zelligen Elementen des durch Generationen fortgezüchteten 'Iumors abstammt, sondern dass sie unter dem chemischen Einfluss von Geschwulstzellen aus normalen Körperzellen entstanden ist. Mithin sind diese Sarkome experimentell erzeugte neue Geschwïlste. Ist freilich die spezifische Umwandlung der Bindegewebszelle in die Sarkomzelle erfolgt, so muss letztere infolge ihrer gesteigerten Vitalität bei der Transplantation nicht mehr zugrunde gehen. Vielmehr verhält sie sich jetzt wie die Karzinomzelle und lässt sich von nun ab ebenfalls in Generationen fortzüchten.

Die Annahme einer von den Tumorzellen ausgehenden geschwulsterregenden Reizwirkung, die u. a. von Coenen anch für die sekundäre Karzinombildung unter dem Einfluss primärer Sarkome akzeptiert worden ist, bildet eine nicht unwichtige Stütze 
Histologische Ergebnisse der experimentellen Krebsforschung. 149

für die in neuerer Zeit als Reaktion gegen die Ribbertsche Lehre von der ausschliesslich unizentrischen Entstehung der Krebse wieder mehr Geltung gewinnende alte Anschauung, dass das expansive Wachstum des Karzinoms auch durch Einbeziehung benachbarten gleichfürmigen Epithels in den krebsigen Prozess stattfinden kann, eine Anschauung, die zwar noch von Borst ${ }^{1}$ ) zurückgewiesen, aber nemerdings von $\mathrm{Lubarsch}{ }^{2}$ ) wieder ernsthaft diskutiert wurde. Ja in weiterem sinne spricht unsere Reiztheorie auch zugunsten der von Waldeyer begründeten mond von den Chirurgen nach Möglichkeit beherzigten Anschauung, dass ein ganzes Organ zum Krebs disponieren kann.

Von $\mathrm{L}$ e w in,$^{3}$ ) der bei seinem Ratten-Adenokarzinon sekundär nicht nur ein Sarkom, sondern auch ein Kankroid auftreten sah, ist unsere Erklärung auch auf letzteres ausgedehnt worden. obwohl in derartigen Fallen die Möglichkeit einer metaphastischen Umwandhng; wie 1 . a. auch $\mathrm{Orth}$ ) vor kurzem bei analogen Erscheitungen in der menschlichen Pathologie betont, nicht ganz ausgeschiossen ist.

Im Hinblick auf diese echten sekundären Sarkome, die also mach unserer Auffassung nur eine kausale, nicht aber eine genetische Beziehung zum Karzinom haben, gewinnen die eigentümlichen Strukturveründerungen, die wir an unserem Karzinomstamm 11 beobachten konnten, ein erhöhtes Interesse.

Dieser Stamm 11 leitet sich von einem primüren Mammatumor einer weissen Vaus $a b$, der dem von mir aufgesteliten Typus des spaltenbildenden liar\%inoms entspricht. Ich verstehe darunter eine Krebsform. bei der die auf den Drüsenursprung der Geschwulst hinweisenden Lumina nicht die regelmässig runde oder ovale Form wie in den einfachen Adenomen haben, sondern vielfach höchst unregelmässig verzerrt und zu engen Spalten ausgezogen erscheinen. Die die Spaltriume auskleidenden Epithelien liegen gewöhnlich mehrschichtig und gehen vielfach mit denen der Nachbarschaft feste strangartige Verbindungen eill. So stellt denn der Tumor ein weitverzweigtes Netz von Karzinombalken und mannigfach gestaltenen Hohlrăumen dar. Diese Krebsform

\footnotetext{
2) Die Lehre von rlen Geschwalstzellen.

2) Verhand. d. deutseb. pathol. Ges, in Kiel. 1908.

a) Zeitschr. f. Krebsforschung, Bd. 6, H. 2. 1907.

$\left.{ }^{4}\right)$ Sitzungsber. d. Königl. Preuss. Akad. d. Wiss. 1909.
} 
neigt zur Bildung papillärer Strukturen, die bei der Maus zwar selten grössere Dimensionen annehmen, und auch im vorliegenden Falle, obwohl deutlich vorhanden, sich in bescheidenen Grenzen halten. Der geschilderte Bau blieb nun bei dem Stamm 11 über viel Generationen in ziemlich unveränderter Weise bestehen. Fig. 2 stammt von einer Geschwulst der 31. Generation und weist

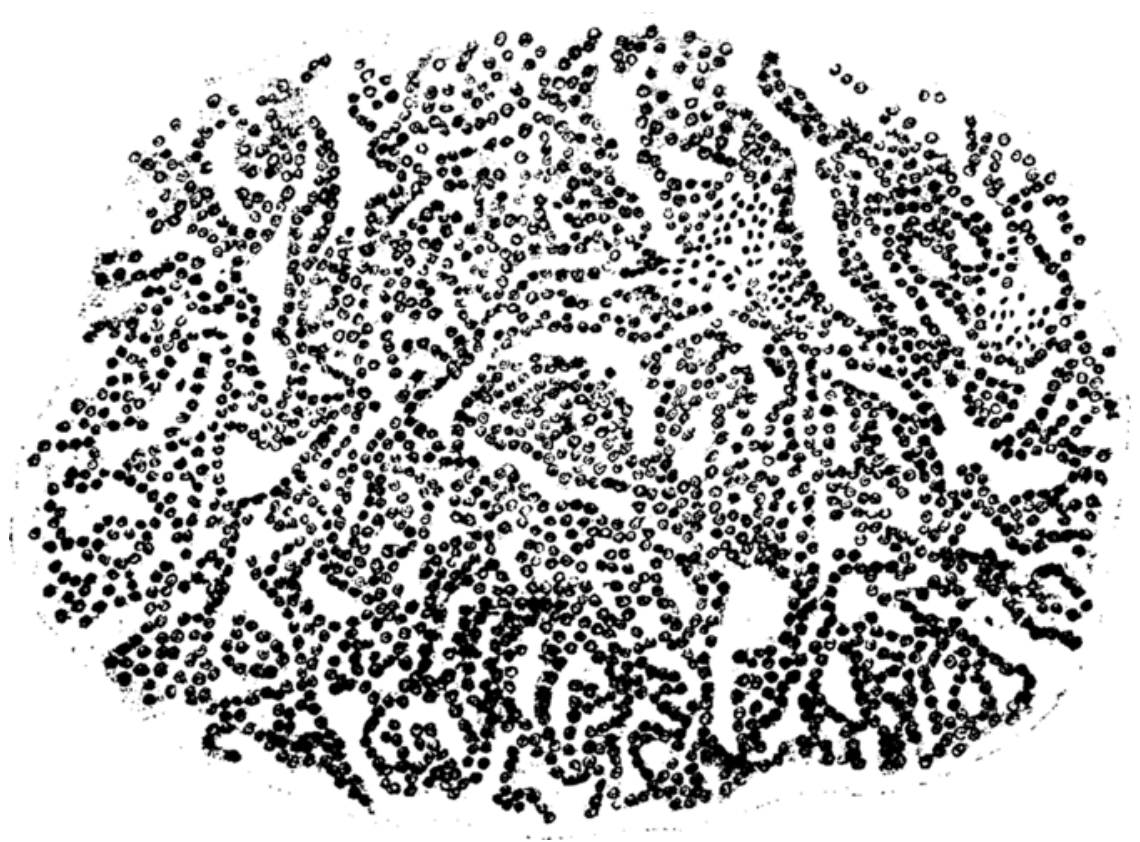

Fig. 2.

vollkommen die gleichen Verhăltnisse wie der Primärtumor auf. Allmählich jedoch machte sich eine Änderung des Wacbstums insofern geltend, als der drüsige Bau, also die Bildung der Lumina abnahm zugunsten solider Nester und Stränge, in denen nur gelegentlich noch die Andeutung eines adenomatösen Baues erkennbar ist. Schliesslich schwindet die letzte Spur einer Lumenbildung, wăhrend der alveoläre Bau in optima forma ausgesprochen ist. Die runden hart aneinander stossenden Alveolen zeigen gewöhnlich ein zentrales Gefäss, so dass in der ganzen Konfiguration eine entfernte Ähnlichkeit mit einem Peritheliom entsteht. $\mathrm{Zu}$ diesen tiefgreifenden Änderungen des Aufbaues tritt nun noch 
ein weiteres Moment, das an sich bekannt und in menschlichen Karzinomen bäufig beschrieben, durch seinen Umfang dem Tumor einen ganz eigenartigen histologischen Charakter verleiht, nämlich eine Umwandlung kubischer Epithel- in ausgesprochene Spindelzellen. Dadurch, dass die Abplattung der Epithelien nach der Peripherie immer mehr zunimmt, und die Zellen hier häufig zwiebelschalenartig angeordnet sind, entsteht eine gewisse Ähnlichkeit der Alveolen mit Hornperlen (Fig. 3). Diese sich leicht aus mechanischen Verhăltnissen erklärende Formveränderung der Zellen wäre an sich nicht so merkwürdig, wenn sie an der Grenze des

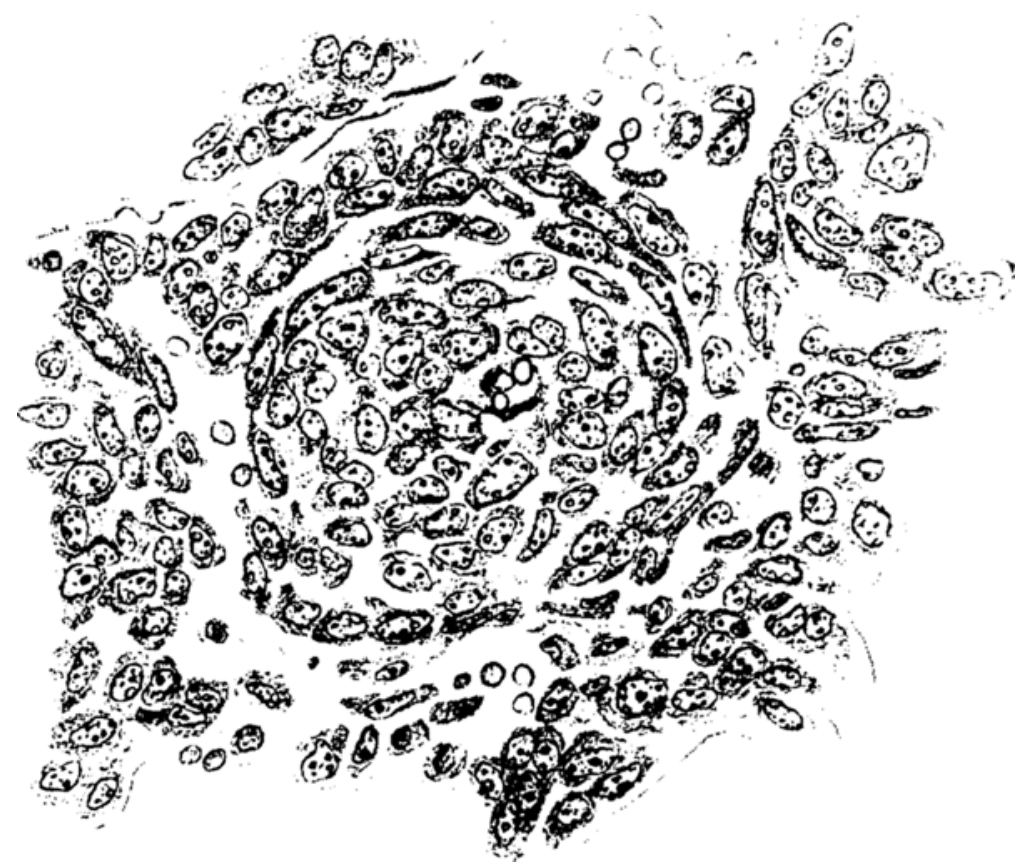

Fig. 3.

Alveolus Halt machte. Dies ist aber nicht der Fall, vielmehr setzt sich die Formation von Spindelzellen auch auf das ganze zwischen den Alveolen liegende Gebiet fort, ohne dass es möglich wäre, irgendwo eine Grenze zu ziehen. Hand in Hand mit dieser Umwandlung der Zellform geht ein auffallender Schwund des Stromas, das vielfach selbst mit den besten Darstellungsmethoden des Bindegewebes kaum in Spuren noch nachzuweisen ist. 
Als ich den Tumor in diesem Stadium in Kiel demonstrierte, hielt ich eine definitive Entscheidung darüber; ob es sich hier nur um Formveränderungen der Karzinomzellen oder um den Beginn einer Sarkomentwicklung handelt, für kaum möglich, neigte aber mehr zu der ersteren Erklärung, vor allem deswegen, weil zwischen den verschiedenen Zellformationen nirgends, wie beim echten Carcinoma sarcomatodes eine Grenze zu ziehen ist. Auch unter den anwesenden Pathologen war die Meinung vorherrschend, dass den Zellveränderungen keine entscheidende Bedeutung zukommt, und der 'Tumor' nach wie vor als Karzinom gedeutet werden muss.

Inzwischen hatte nun der Prozess weitere Dimensionen angenommen, die die Entscheidung immer schwieriger gestalteten. Es kamen häufig Tumoren zur Untersuchung, in denen auf weite

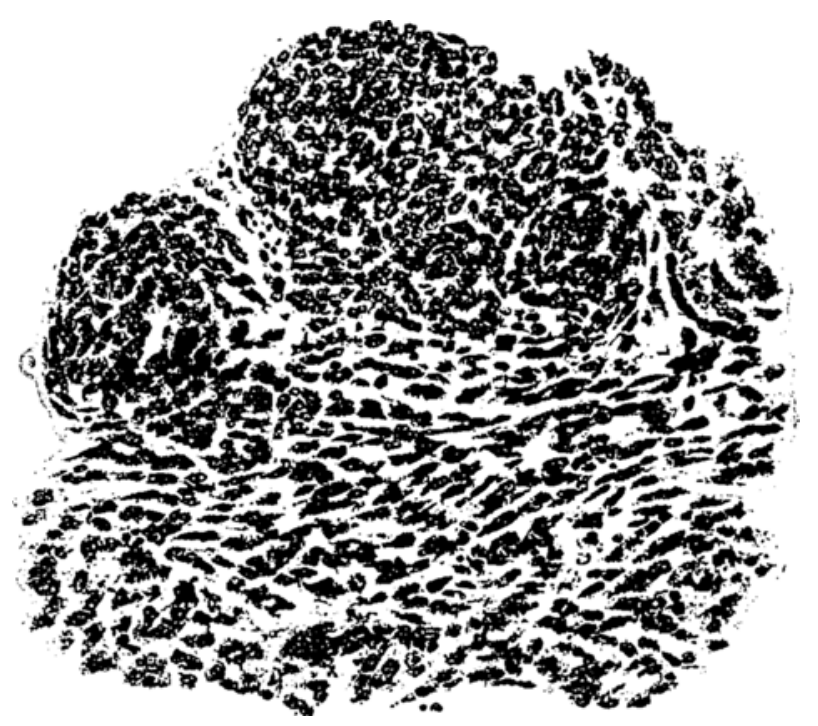

Fig. 4.

Strecken der alveoläre Bau nur eben angedeutet war, während im übrigen die Zellen ziemlich diffus verteilt überall in spindlige Formationen übergingen. Die Fig. 4 und j geben von diesen Verhaltnissen eine recht gute Vorstellung. Dabei möchte ich besonders auf den in den Abbildungen deutlich wahrnehmbaren Umstand hinweisen, dass die Spindelzellen keineswegs dicht ge- 
drängt und gegeneinander abgeplattet, sondern im Gegenteil ziemlich frei und isoliert liegen, so dass das sonderbare Verhälnis mit mechanischen Ursachen nicht erklärt wird. Dazu kam nun ein weiteres, ebenfalls aus Fig. 5 ersichtliches Moment, numlich das gelegentliche Auftreten von Tumorriesenzellen, das auf einen Kampf des allmählich untergehenden Karzinoms gegen das überwuchernde spindlige Gewebe zu deuten schien. Ich kann nicht leugnen, dass es mir in diesem Stadium ausser-

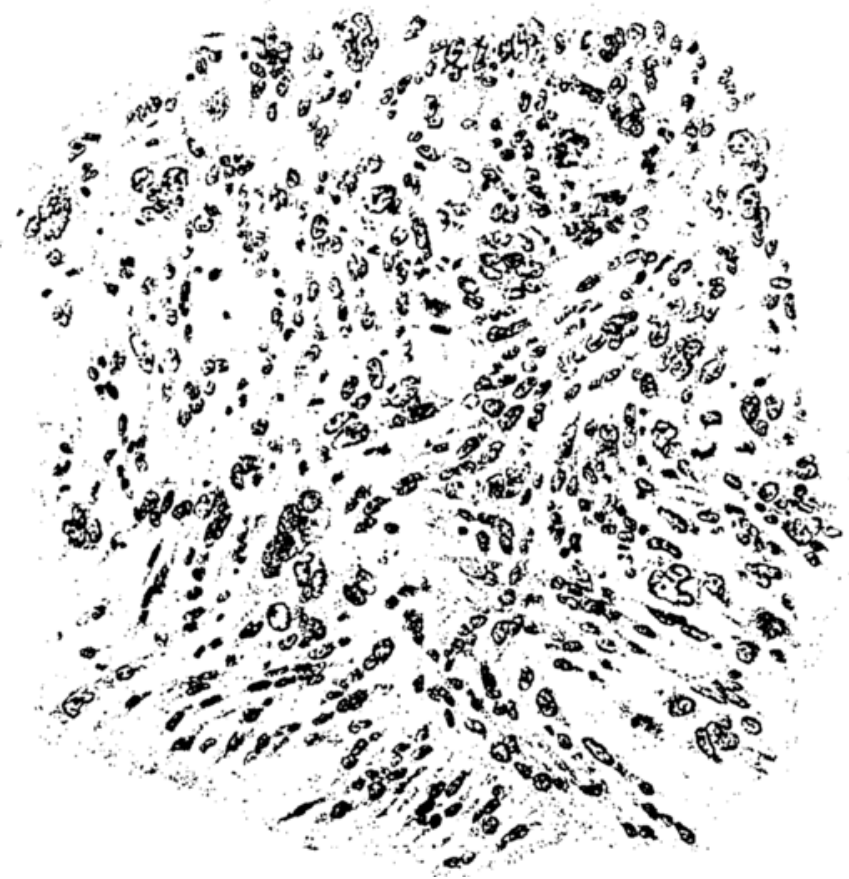

Fig. 5 .

ordentlich schwer geworden ist, noch an die Karzinomnatur des 'Tumors zu glauben, und dass ich in diesem Fall von der metaplastischen Umwandlung der Karzinom- in die Sarkomzelle fest überzeugt gewesen wäre, wenn nicht der karzinomatöse Charakter in der Bildung von Nestern und Strängen immer wieder hervorgetreten wïre und allmählich sogar von neuem das Übergewicht erreicht hatte. Schliesslich musste jeder Zweifel schwinden, als plötzlich in der 139. Generation abermals eine durchgreifende 
strukturelle Veränderung Platz griff. Wie mit einem Schlage waren alle spindligen Formationen geschwunden, und die Geschwulst präsentierte sich wieder als ein typisches Adenokarzinom unter Bildung deutlicher Lumina, die sich auch da nicht ganz verlor, wo die Zellen zu grösseren Balken zusammentraten (Fig. 6).

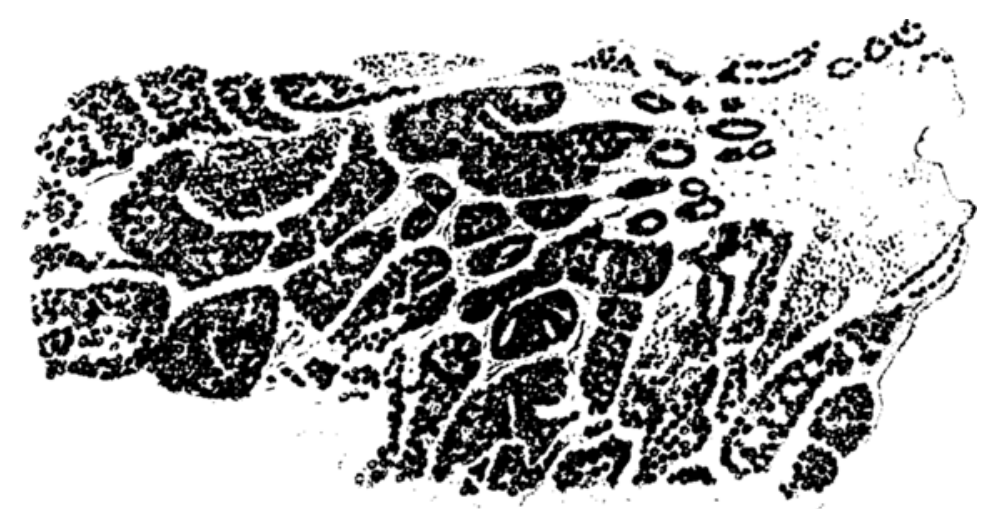

Fig. 6.

Dieser abermalige Strukturwechsel war ein so plötzlicher. dass man im ersten Augenblick an die Möglichkeit einer Tumorverwechslung denken musste. Indessen zeigte, abgesehen davon, dass die Einrichtung unseres Betriebes diese Annahme wenig wahrscheinlich machte, ein genaueres Nachforschen, dass sie direkt auszuschliessen ist. Neue Serien werden bei uns gewöhnlich so angelegt. dass ein, zwei oder drei Tumoren aus einer oder mehreren Serien für sich oder gemischt auf 30 Tiere verimpft werden. Es kommt daher hăufig vor, dass mit verschiedenen Iumoren einer Serie verschiedene neue Serien angelegt werden, aber nie, dass ein Tumor zur Impfung verschiedener Serien verwandt wird. Nun ergab die Untersuchung, wie untenstehend skizziert, folgendes:

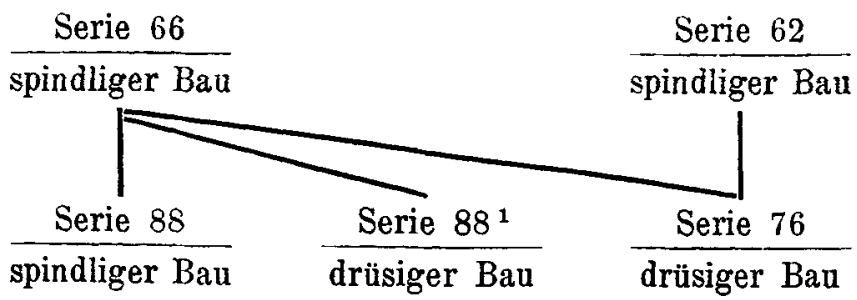


Von Serie $66^{1}$ ) leiten sich drei Serien her, Serie $88,88^{1}$ und 76. Erstere zeigt, ebenso wie die Mutterserie 66 spindlige Formation, Serie $88^{1}$ dagegen und Serie 76 Drüsenkarzinom. Zur Herstellung der Serie 76 diente ausserdem die ebenfalls spindelförmig gebaute Serie 62. Läge eine Verwechslung vor, so müsste sich dieselbe mindestens auf zwei Tiere der Serie 66 beziehen, da ja für die Serien $88^{1}$ und 76 verschiedene Tiere der Serie 66 verwandt wurden. Das ist aber so gut wie auszuschliessen. Hierzu kommt nun noch ein weiterer innerer Grund. Ich konnte seinerzeit an zahlreichen Tumormischungsversuchen den Nachweis führen, dass bei der Mischung verschieden virulenter Geschwülste die weniger virulenten stets von den virulenteren iiberwuchert werden. Nun unterliegt es aber auf Grund des makroskopischen Wachstums und des histologischen Mitosennachweises nicht dem geringsten Zweifel, dass der Tumor im Stadium der spindligen Struktur weitaus die grösste Virulenz aufwies. l)a aber für den Fall einer Verwechslung Serie 76 aus der Mischung eines spindligen (Serie 62) und eines Drüsenkarzinoms (Serie 66) entstanden ware, so hatte sie unbedingt wieder den spindligen Charakter aufweisen müssen. Die Strukturveränderung ist nicht anders als mit irgendwelchen nicht weiter definierbaren biologischen Änderungen der Tumorzelle selbst zu erklüren, die gewissermassen einen Rückschlag in ein früheres Stadium bedeuten und jedenfalls beweisen, dass die Geschwulst trotz ihrer merkwürdigen histologischen Variationen nie aufgehört hat, ein Karzinom zu sein. Die Plötzlichkeit dieser vitalen Zelländerung steht nicht obne Analogon. Hatten wir doch bei einer unserer echten Sarkomumwandlungen ebenfalls eine plötzliche Änderung von einer Generation zur folgenden beobachtet.

Diejenigen Autoren, die wie v. Hansemann die endotheliale Natur der Mäusetumoren zu vertreten geneigt sind, werden vielleicht in den beschriebenen Metamorphosen eine Bestätigung ibrer Anschauung erblicken. Demgegenüber kann nicht scharf genug betont werden, dass mit Ausnahme einer geringen Anzahl in den letzten Jahren bekannt gewordener anderer Typen wie Cancroid, Chondrom, Lymphosarkom etc., die zusammen sicherlich nicht mehr als $3 \%$ aller hier vorkommender Tumoren

) Die Zahlen bedeuten den Tag des Jahres, an dem die betreffende Serie angelegt ist. 
ausmachen, das Gros der Mäusegeschwülste epitheliale Mammatumoren sind. Wenn Deton ${ }^{1}$ ) vor kurzem gegen diesen von mir erbrachten und fast allseitig bestätigten Nachweis einwendet, dass in zwei von ihm mit der Plattenmodelliermethode untersuchten Fällen ein Zusammenhang der Tumoren mit der in der Năhe gelegenen Brustdrüse nicht zu konstatieren war, so ist darauf zu erwidern, dass die angewandte Methode mit ilıren unvermeidlichen Fehlerquellen für die hier in Betracht kommenden Verbältnisse nicht ausreicht. Hält es doch selbst bei der normalen Namma oft schwer, einen Zusammenhang zwischen den scheinbar isoliert im Fettgewebe gelegenen Acini zu erkennen.

Eine eingehendere Schilderung der an unserem Karzinomstamm 11 beobachteten Strukturveranderungen schien mir deswegen geboten, weil dieselben in ihrem Umfang und zyklischen Verlauf unzweifelhaft ein seltenes Vorkommnis darstellen, und weil sie deutlich zeigen, dass auch bei den Mausetumoren die durch die klassischen Untersuchungen von Thie rsch und Waldeyer fest begründete Differenz zwischen der Genese der Karzinome und der der Sarkome in vollem Umfang zu Recht besteht.

Bei der vielfach in Schrift und Wort offenkundig zum Ausdruck kommenden Neigung, auf Grund der experimentell erforschten Sarkomentwicklung einen innigeren Zusammenbang zwischen den Sarkomen und Karzinomen zu statuieren, als es den überkommenen Anschauungen entspricht, lege ich Wert darauf und möchte es durch die vorstehende Mitteilung erhärtet wissen, dass auch eine weitgehende histologische Annăherung der beiden Geschwulsttypen an ihrer prinzipiellen Trennung nichts andert.

1) Zeitschr. f. Krebsforsch., Bd. 8, H. 3. 1910. 